\title{
Tomographic Reconstruction of the Low-Latitude Nighttime Electron Density Using FORMOSAT-3/COMSIC Radio Occultation and UV Photometer Data
}

\author{
Kenneth F. Dymond ${ }^{1, *}$, Scott A. Budzien ${ }^{1}$, Damien H. Chua ${ }^{1}$, \\ Clayton Coker ${ }^{1}$, and Jann-Yenq Liu ${ }^{2}$ \\ ${ }^{1}$ Space Science Division, Naval Research Laboratory, Washington, District of Columbia, USA \\ ${ }^{2}$ Institute of Space Science, National Central University, Chung-Li, Taiwan, ROC
}

Received 16 June 2007, accepted 15 January 2008

\begin{abstract}
The Constellation Observing System for Meteorology, Ionosphere, and Climate (FORMOSAT-3/COSMIC) is a constellation of six microsatellites that was launched into low-Earth orbit on 14 April 2006. Each FORMOSAT-3/COSMIC satellite contains a GPS Occultation Experiment (GOX) GPS receiver and a Tiny Ionospheric Photometer (TIP), which measure the ionosphere. In previous papers of Dymond and Thomas (2001) and Dymond et al. (2000), an algorithm for tomographically inverting GPS occultation and UV radiometer measurements has been presented. We apply this algorithm to the inversion of recently acquired FORMOSAT-3/COSMIC data and present the results.
\end{abstract}

Key words: Ionosphere, Tomography, FORMOSAT-3/COSMIC, Appelton anomaly

Citation: Dymond, K. F., S. A. Budzien, D. H. Chua, C. Coker, and J. Y. Liu, 2009: Tomographic reconstruction of the low-latitude nighttime electron density using FORMOSAT-3/COMSIC radio occultation and UV photometer data. Terr. Atmos. Ocean. Sci., 20, 215-226, doi: 10.3319/TAO.2008. 01.15.01(F3C)

\section{INTRODUCTION}

The use of total electron content measurements acquired during occultation of the Global Positioning Satellites (GPS) by a receiving platform in low Earth orbit has recently been demonstrated as a viable technique for determining the ionospheric electron density (Hajj et al. 1994). The cornerstone of the technique is use of the Abel inversion to convert the total electron content measurements made by the GPS receiver into electron density profiles. This technique assumes that the ionosphere is spherically symmetric with no horizontal density gradients. The Abel technique works well under certain viewing conditions but its accuracy is generally limited by the spherical symmetry assumption. When there are substantial ionospheric density gradients present, the Abel inversion technique yields poor results due to an inability to handle gradients (Hajj et al. 1994). As GPS occultations occur sporadically around the globe, it is difficult to use them in a systematic way to gather information about the global structure of the ionosphere. Thus, alternative tech-

\footnotetext{
* Corresponding author

E-mail: Kenneth.dymond@nrl.navy.mil
}

niques must be used to infer the gradients and to account for them in the inversion process.

Several techniques have been used to infer ionospheric gradients. On the Formosa Satellite -3 or Constellation Observing System for Meteorology, Ionosphere, and Climate (FORMOSAT-3/COSMIC), GPS occultation measurements will be complemented by ultraviolet radiometric measurements, measurements of the total electron content between the satellites and ground using computerized ionospheric tomography receivers, and ground-based GPS total electron content measurements. The use of ultraviolet (UV) radiometers provides an accurate measurement of the gradients on a global basis and does not rely on ground-based receivers.

Naturally occurring ultraviolet and visible emissions from the night sky have been used since the late 1960's as means of determining the densities of electrons and $\mathrm{O}^{+}$ions in the F-region ionosphere. The emissions are produced by radiative recombination of $\mathrm{O}^{+}$ions and electrons to produce atomic oxygen in an excited state that subsequently decays by emitting a photon. These emissions have been observed 
at visible wavelengths (7774 and $8446 \AA$ ) from the ground (Tinsley et al. 1973; Tinsley and Bittencourt 1975) and at ultraviolet wavelengths $(911,1304$, and $1356 \AA)$ from space (Barth and Shaffner 1970; Hicks and Chubb 1970; Chakrabarti et al. 1984; Feldman et al. 1992). The use of nighttime $\mathrm{O}$ emissions to remotely sense the F-region electron density is described by Chandra et al. (1975), Tinsley and Bittencourt (1975), Meier (1991), and Dymond et al. (1996, 1997). UV emissions are of most interest for space based ionospheric sensing. Earth's atmosphere absorbs all UV emissions, with wavelengths less than $1800 \AA$, at an altitude of approximately $90 \mathrm{~km}$. Thus, there is no UV background. Earth's atmosphere is transparent to visible emissions and, therefore, there is a visible background due to city lights, forest fires, thunderstorms, noctilucent clouds, and reflection of moon light; these background emissions would require measurement and subtraction from the nightglow sig- nal. This background subtraction can lead to large uncertainties in the derived airglow signal. The problems caused by background emission are far worse on the dayside because the background emissions can originate above $100 \mathrm{~km}$.

We present the results of tomographic inversions of the TIP radiances in conjunction with the GOX occultation TECs. The occultations occurred in the region of the Appelton anomalies where the ionospheric gradients are very large. Figure 1a shows the distribution of the measured $135.6 \mathrm{~nm}$ ionospheric radiances from all six TIP instruments during a single day. Figure $1 \mathrm{~b}$ shows the $135.6 \mathrm{~nm}$ radiance calculated from the electron densities obtained from the GOX Abel inversions. The TIP measurements clearly better characterize the structure of the ionosphere in and near the Appelton anomalies, while the occultations tend to smear out the structure. This is due to the fact that the GOX Abel inversions assume spherical symmetry of the ionosphere,
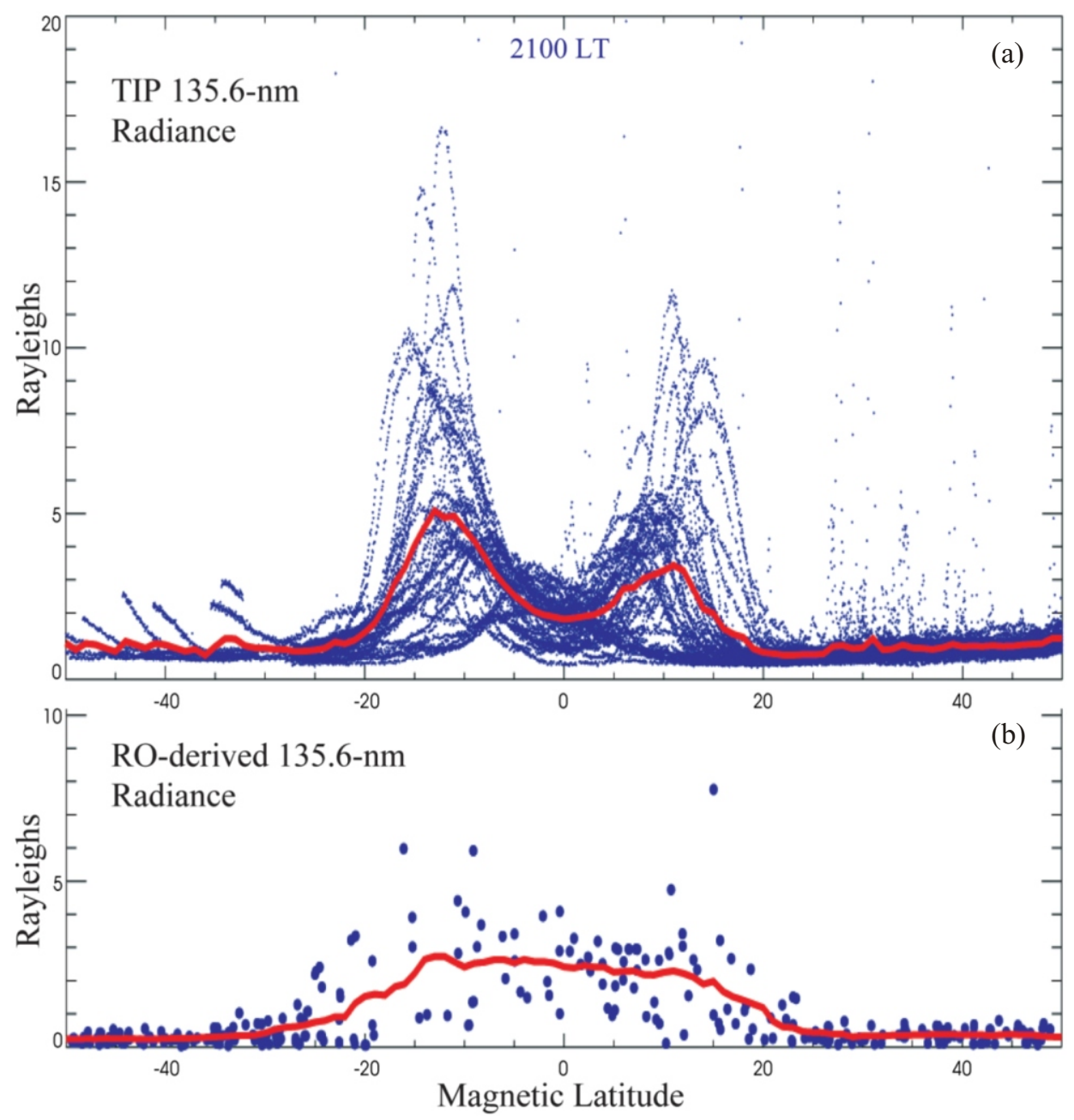

Fig. 1. Comparison of TIP and GOX morphologies near the equatorial anomalies. The solid line indicates TIP radiance averaged over one day and all geomagnetic longitudes and the smaller dots indicate the individual TIP measurements that went into the calculation of the average. The large variation is due to the longitudinal variability of the ionosphere as the measurements uncertainties in the TIP data are $<3 \%$. The dots in (b) indicate one day's worth TIP radiances derived by converting the GOX electron density profiles into volume emission rates and then integrating vertically. Note that the GOX occultations do not adequately capture the morphology in the region of the equatorial anomalies. 
which is clearly not a good assumption in the low-latitude ionosphere. We show that tomographic inversions yield results that differ from Abel inversions as expected and discuss the results.

In this paper, we first present a description of the Tiny Ionospheric Photometer. This is followed by a discussion of the TIP measurements. We then introduce the inversion algorithm and the modifications that have been made since previous publications discussing the technique. The results of the inversions are presented and discussed. Lastly, this work is summarized. Additional information about the TIP instruments and their ionospheric measurements can be found in Chua et al. (2009), Coker et al. (2009), and Tsai et al. (2009).

\section{TINY IONOSPHERIC PHOTOMETER}

The TIP instrument for the FORMOSAT-3/COSMIC satellites has to provide accurate and precise measurements of the electron density gradients in the ionosphere and compliment GOX TEC measurements, which are of very high precision. The instrument also had to be a compact, lightweight package such that it would not tax satellite resources too greatly. We opted to design the system based on measurement of the O I $135.6 \mathrm{~nm}$ emission produced by radiative recombination of $\mathrm{O}^{+}$ions and electrons, a natural decay process of the ionosphere. In order to perform tomographic inversion of the TIP and GOX data simultaneously, the data sets should have comparable signal-to-noise ratios. However, this is difficult to achieve for a compact optical instrument as the airglow signals are not very bright and the small size of the instrument limits the size of the telescope. The design selected was a compact narrow band ultraviolet photometer. Additional details of the instrument and its design can be found in Kalmanson et al. (2004); we summarize the relevant design features here.

The TIP instrument is a narrow band photometer that measures the $135.6 \mathrm{~nm}$ emission over the passband of 132.5 to $160 \mathrm{~nm}$. The instrument is divided into two sections: the interface electronics box and the photometer head, as shown in Fig. 2. The interface electronics provides interface to the space vehicle and is the control system for the TIP instrument. The photometer subsystem consists of an off-axis parabolic telescope feeding a sub-miniature Hamamatsu photomultiplier tube with a cesium iodide photocathode. The photometer includes a filter wheel assembly with additional filters and a shutter to block the light path so that the on-orbit radiation background seen by the photomultiplier can be measured. The wavelength passband of the system is controlled by the filter and photocathode combination.

The short wavelength edge of the passband is determined by the short wavelength cut-off of the strontium fluoride filter placed in the light path. Strontium fluoride has a short wavelength cut-off at $\sim 128 \mathrm{~nm}$ at room temperature (Samson 1967); no light at wavelengths shorter than this is transmitted through the filter. When the filter temperature is increased to $100^{\circ} \mathrm{C}$, the cut-off shifts from 128 to $132.5 \mathrm{~nm}$; this effectively eliminates the nearby emission from $130.4 \mathrm{~nm}$, which is also produced by radiative recombination of $\mathrm{O}^{+}$and electrons. The $130.4 \mathrm{~nm}$ emission is difficult to interpret because the vertical optical depth of this emission reaches several thousands at the bottom of the F-region ionosphere; thus, it is not a good candidate for ionospheric monitoring. The $135.6 \mathrm{~nm}$ emission is largely optically thin in the ionosphere and thus is the emission selected for the observations. The long-wavelength cut-off of the TIP passband is determined by the fall-off of the quantum efficiency of the cesium iodide photocathode of the photomultiplier tube. The longwavelength cut-off of cesium iodide is nominally at about $160 \mathrm{~nm}$. However, examination of the TIP data during normal orbit operations indicated that the TIP data showed the presence of city lights. This is unfortunate because it means that the long wavelength sensitivity of the TIPs is much higher than expected. This is due to contamination of the cesium iodide photocathode by other metals having lower photoelectric work function; this contamination occurred during the preparation of the photomultiplier tube at Hamamatsu. The large light grasp of the instrument compounded the effects of the long wavelength sensitivity causing visible emissions from the ground to be detectable.

An instrument's sensitivity is defined as the number of photons detected per second at an input radiance of 1 Rayleigh:

$S=\frac{10^{6}}{4 \pi} Q T R A \Omega$

where $Q$ is the quantum efficiency of the photocathode on

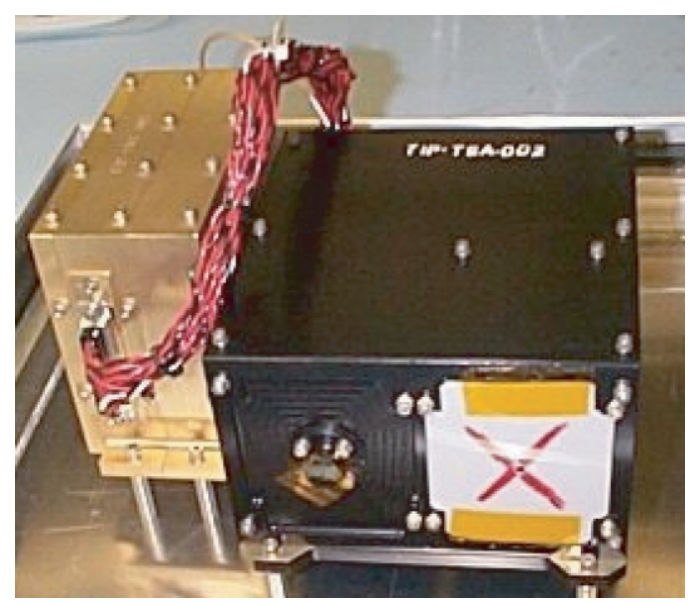

Fig. 2. TIP Instrument. This is a photograph of the TIP instrument. The photometer head is the black box and the electronics and interface box is the gold box. 
the detector (number of photons detected/number of photons incident), $T$ is the filter transmittance, $R$ is the reflectance of the telescope, $A$ is the collecting area of the telescope in $\mathrm{cm}^{2}$, and $\Omega$ is the instrument's field-of-view in steradians. The factor $10^{6} / 4 \pi$ converts the radiance from units of Rayleighs to photons s $\mathrm{sm}^{-1}$ steradian $^{-1}$ (the $4 \pi$ factor is required here to convert from the omnidirectional radiance, expressed in Rayleighs, to a specific radiance per steradian). The field-of-view is $3.8^{\circ}$ and is circular; this was driven by the size of the photocathodes available for the photomultiplier tube and by the desired scale sizes we wished to observe. This field-of-view provides a best-case spatial resolution at the F-region peak $(\sim 350 \mathrm{~km})$ of $\sim 11 \mathrm{~km}$ when the satellites are in their initial orbit at $\sim 515 \mathrm{~km}$ altitude and $\sim 30 \mathrm{~km}$ when the satellites are at their nominal orbit altitude of $\sim 800 \mathrm{~km}$. This spatial resolution is smeared out along the orbit track by an additional $8.3 \mathrm{~km}$ due to the satellite's orbital motion and the nominal 1.18 second integration time for the TIP measurements. Using measured values for the quantum efficiency and the filter transmittance and the known values for the reflectance and area of the telescope mirror, the TIP sensitivity was estimated to be $\sim 500 \mathrm{ct} \mathrm{s}^{-1}$ Rayleigh $^{-1}$.

\section{ALGORITHM}

\subsection{Description}

We developed and tested an algorithm that simultaneously inverts the TIP vertical radiance measurements and the GPS occultation data to produce a high fidelity ionospheric reconstruction along the orbit track using a tomographic scheme, as seen in our previous work: Dymond et al. (2000), Dymond and Thomas (2001). The GPS total electron data are in the form of an occultation or "limb scan" and therefore these data provide high quality altitude information. However, the GPS data are integrated electron densities along the line-of-sight to the GPS satellite and therefore may be affected by gradients along the line-of-sight. The intensity measured by the radiometer is proportional to the vertical column integral of the square of the electron density and, therefore, contains no altitude information. But, the radiometer data contains high quality gradient information. The use of the two data sets together offsets the weaknesses in each data set while taking advantage of their strengths.

The nighttime $135.6 \mathrm{~nm}$ emission shows contributions due to radiative recombination and $\mathrm{O}^{+}-\mathrm{O}^{-}$neutralization (Tinsley and Bittencourt 1975). The emission also shows mild opacity effects due to multiple scattering of the radiation by atomic oxygen in the thermosphere. The emission is also a doublet emission originating in a singlet upper state with wavelengths 135.6 and $135.8 \mathrm{~nm}$; the doublet is not resolved by the TIP. The volume emission rate, $\varepsilon_{0}(z)$, for the emission is given by (Tinsley and Bittencourt 1975):

$$
\begin{aligned}
\varepsilon_{0}(z)= & \gamma \beta_{1356} \frac{k_{1} k_{2} n_{e}(z) n_{O}(z) n_{O^{+}}(z)}{k_{2} n_{O^{+}}(z)+k_{3} n_{O}(z)} \\
& +\gamma \alpha_{1356} n_{e}(z) n_{O^{+}}(z)
\end{aligned}
$$

where $\gamma$ is the branching ratio for the 1356 or $1358 \AA$ line [0.791 and 0.209, respectively, (Meier 1991)], $\alpha_{1356}$ is the radiative recombination rate $7.5 \times 10^{-13} \mathrm{~cm}^{-3} \mathrm{~s}^{-1}$ (Melendez-Alvira et al. 1999), $\beta_{1356}$ is the fraction of neutralizations that produce atoms in the ${ }^{5} \mathrm{~S}$ state, 0.54 , and the coefficients $k_{1} k_{2}$, and $k_{3}$ are $1.3 \times 10^{-15}, 1.0 \times 10^{-7}$, and $1.4 \times$ $10^{-10}$, respectively, all in units of $\mathrm{cm}^{3} \mathrm{~s}^{-1}$ (Tinsley and Bittencourt 1975). To simplify the calculations, we make the assumption that the first term in Eq. (2) can be ignored. Furthermore, the $135.6 \mathrm{~nm}$ emission shows mild opacity and radiation transport effects which we also neglect as the contribution to the $135.6 \mathrm{~nm}$ radiance is generally small. Thus, the $135.6 \mathrm{~nm}$ radiance is given by:

$4 \pi I=10^{-6} \int_{0}^{\infty} \varepsilon_{0}(z) d z$

where the factor $4 \pi$ indicates that the radiance indicates the number of photons emitted into $4 \pi$ steradians and the factor of $10^{-6}$ converts from photons to megaphotons, the natural unit for Rayleighs.

The GPS receiver measures the total electron content given by:

$\operatorname{TEC}(\varphi)=\int_{0}^{\infty} n_{e}[z(s)] d s$

where $n_{e}$ is the electron density at altitude $z$ and $d s$ is the differential path length from the observer.

The altitude distribution of the $\mathrm{O}^{+}$and electron density was parameterized using a generalized Chapman layer (Chamberlain and Hunten 1987). We used five parameters to characterize the ionosphere: the altitude where the density peaks, $h m F 2$; the density at the peak, $n m F 2$; and three parameters to characterize the scale height, $H(z)$, which is onehalf the plasma scale height for the form of the Chapman layer given below. The Chapman function for describing the $\mathrm{O}^{+}$density, $n_{O^{+}}(z)$, is:

$n_{O^{+}}(z)=n m F 2 \exp \left\langle\frac{1}{2}\left\{1-\frac{z-h m F 2}{H(z)}-\exp \left[-\frac{z-h m F 2}{H(z)}\right]\right\}\right\rangle$

where $z$ is the altitude. The scale height is assumed to be a constant below $h m F 2$ and to vary quadratically with the difference in altitude above the peak: $H(z)=H_{0}+H_{l}(z-$ 
$h m F 2)+H_{2}(z-h m F 2)^{2}$, where $H_{0}, H_{1}$, and $H_{2}$ are parameters. This form of the Chapman layer is an extension to the linearly varying scale height proposed by Picone et al. (1997) and is a reasonable approximation for describing the ionospheric density. The $\mathrm{O}^{+}$and electron densities are assumed to be equal, which is a good assumption below the $\mathrm{H}^{+} / \mathrm{O}^{+}$transition height that typically occurs at $\sim 750 \mathrm{~km}$.

The along-track variation of the electron density is parameterized by placing Chapman profiles spaced every degree along the orbit track. The profiles are all uncoupled and no smoothing or regularization is used; this causes the inversion to be more sensitive to point to point variations in the photometer data. Some of the point-to-point variation is due to photon shot noise on the TIP measurements; some of the variation is due to ionospheric structure. The same altitude variation is assumed for all of the profiles, as the altitude variation is determined at one location near the tangent point locus of the GPS occultation. The individual profiles are scaled by $n m F 2$ to characterize the along track electron density variations. The $n m F 2$ variation between the profiles is given by a quadratic Lagrange interpolation polynomial (Press et al. 1992). These are reasonable approximations to make as the GPS occultations provide accurate altitude distribution information while the photometer data provide accurate information on $n m F 2$ and no information about the altitude distribution. However, both the scale height and peak height of the electrons are known to vary with latitude in the ionosphere.

The line-of-sight of a GPS occultation spans $56^{\circ}$ of latitude from a satellite at an altitude of $800 \mathrm{~km}$ when the tangent altitude of the line-of-sight is $100 \mathrm{~km}$. Thus, our model ionosphere may have 56 parameters to describe the along-track $n m F 2$ variation and 4 additional parameters to describe the shape of the profiles.

The inversion algorithm uses an iterative approach, based on Discrete Inverse Theory (Menke 1989), to seek the maximum likelihood estimate (minimum of the chi-squared statistic) of the ionospheric parameters based on the fit of the model to the data. The DIT approach starts by estimating the "data" based on the current parameter values. The $\mathrm{O}^{+}$densities are used to calculate the intensity seen by the photometer and the total electron content measured by the GPS receiver. The $\chi^{2}$ statistic (Bevington 1969) is then calculated and used to compare the model to the "data". If the fit is deemed to be acceptable, the algorithm terminates and calculates the "best fit" ionosphere; otherwise, new values for the model parameters are chosen and the whole process is repeated. A fit is considered to be acceptable when the $\chi^{2}$ changes by less than $0.1 \%$ between steps. Since the radiative recombination emissions are proportional to the square of the electron density, the algorithm is non-linear. The parameters are iteratively adjusted using the Levenberg-Marquardt scheme (Press et al. 1992) to seek the minimum $\chi^{2}$. The Levenberg-Marquardt scheme uses partial derivatives of the $\chi^{2}$ with respect to the Chapman parameters in Eq. (5), the scale height parameters, the $n m F 2$ at each of the longitude cells in the reconstruction, the biases, and instrument sensitivity (see discussion below) to estimate the parameter values that will minimize the $\chi^{2}$. The Levenberg-Marquardt algorithm traverses the parameter space along the steepest gradient, like the Steepest Descent algorithm, when the parameters are far from optimal and smoothly transitions to the Inverse Hessian method, which converges quadratically when the parameters are nearing optimality (Press et al. 1992). The partial derivatives of $\chi^{2}$ are estimated using the forward derivative at each step; we have found that in practice this approach does not introduce any errors or affect algorithm convergence. In addition to the ionospheric parameters that obtained the "best fit", the uncertainties in those parameters are also estimated, based on the partial derivatives, and outputted. Errors in the retrieved densities are calculated from the best fit parameters and their uncertainties using conventional error propagation techniques (Bevington 1969).

\subsection{Algorithm Modifications}

There are some modifications to the algorithm that have been made since the original publication in Dymond et al. (2000) and Dymond and Thomas (2001). The original model assumed the $n m F 2$ variation with geographic latitude. While this is an adequate approximation, the variation is better expressed in terms of geomagnetic latitude, so we have modified the algorithm to carry out all calculations in geomagnetic coordinates. The original algorithm also assumed that the GPS lines-of-sight would be projected from their true orientations onto the orbit plane. The current algorithm no longer projects the lines-of-sight onto the orbit plane, rather the calculations are all carried out in geomagnetic coordinates. The only assumption is that the ionosphere does not vary with geomagnetic longitude. This assumption was also implicit in the earlier version of the algorithm; this is why the GOX lines-of-sight were projected onto the orbit plane. The conversion of the algorithm from geographic to geomagnetic coordinates also permits the technique to be used with GPS occultations that are far from the orbit plane. The previous version of the algorithm was limited to using GPS occultations that were within $\sim 20^{\circ}$ of the orbit plane.

Tikhonov (1963) regularization has also been implemented. This permits the algorithm to use data that have lower signal-to-noise ratio but still permits the algorithm to retrieve a smooth, more physical solution. However, the results reported here required a regularization weight of zero, so they are not regularized.

We have also allowed for biases in the data. The GPS data may have receiver and GPS satellite biases present. The TIP data may have a background due to scattered light or particle radiation. In the data set considered in this work, the TIP was in the open aperture mode where the filter wheel did 
not shutter the light so the radiation background could not be assessed. Two additional model parameters were added to account for constant biases: one for the TIP data and one for the GOX data.

The TIP instrument's calibration is expected to degrade during on-orbit operations due to aging of the photocathode. The baseline mode of operations for the instruments was to assume that the calibration degradation could be monitored by recalibrating based on incoherent scatter radar (ISR) overflights. The idea is to measure the $\mathrm{O}^{+}$and electron densities during the ISR overflight and to calculate the expected $135.6 \mathrm{~nm}$ emission based on the ISR measurement. The TIP sensitivity can be calculated by dividing the measured TIP count rate by the ISR emission estimate. We realized that the GPS occultations also provide another means for absolute calibration. The TIP measurements provide the electron density gradients and the GOX measurements provide an absolute measure of the slant TEC. The measured TIP radiance is proportional to the product of the square of the peak electron density and the plasma scale height. The GOX data give estimates of both the peak electron density and the scale height once the electron density gradients are accounted for using the TIP data. Thus, the TIP calibration can be determined during the tomographic fitting process. We added a scalar to convert the TIP count rate into Rayleighs; this is just the instrument sensitivity.

These refinements have made the algorithm more general, more robust, and more accurate than the previous version presented in Dymond et al. (2000) and Dymond and Thomas (2001).

\subsection{Measurement Uncertainties}

The uncertainties in the data are used in chi-squared fitting to determine the relative significance of the data points. The uncertainties in the TIP data were just the square roots of the numbers of counts in each measurement, as the TIP data are photon shot noise limited. The shot noise limit assumption is valid because the photometers count photons and have negligible dark count rates. The uncertainties in the TEC data are assumed to be very small $\sim 0.5$ TECU. This means that the GOX TECs were measured to $\sim 0.5 \%$ accuracy near the peak. This uncertainty is much larger than the uncertainty of 0.003 TECU cited by Hajj et al. (1994). (A TECU or total electron content unit is defined to be $10^{16}$ electrons $\mathrm{m}^{-2}$. The nighttime limb viewing TEC is on the order of 100 TECU, during the GPS occultation measurements made by the FORMOSAT-3/COSMIC GPS receiver.) The larger uncertainty allows us to more equally weight the two measurements sets in the tomographic inversion. If the very small error bars cited by Hajj et al. (1994) are used, the inversions are driven solely by the GOX data and the TIP gradient measurements have no significance. The resulting fit to the TIP data is very poor while, the GOX data are fitted very well. If, on the other hand, the GOX error bars are assumed to be too large say $~ 10$ TECU, then the TIP measurements drive the inversion and the fit to the GOX TECs is very poor. The choice we made enforces the balance between the data sets. The choice of a constant error, independent of altitude, causes the GOX TECs near the peak to be more heavily weighted than the TECs at the top and bottom of the profile. The TECs near the top and bottom of the profile are more strongly affected by electron density gradients that are either between the observer and the tangent point (foreground electron density) or between the tangent point and infinity (background electron density) than the TECs measured near the peak.

\section{RESULTS AND DISCUSSION}

We consider two sets of observations made by TIP unit 6 on FORMOSAT-3/COSMIC satellite 6 near 2100 LT 15 September 2006 over the Pacific Ocean. The $10.7 \mathrm{~cm}$ solar radio flux $81 \times 10^{-22} \mathrm{~W} \mathrm{~m}^{-2} \mathrm{~Hz}^{-1}$ and the three-hour $\mathrm{a}_{\mathrm{p}}$ ranged from 0 - 2 indicating quiet geomagnetic conditions at the minimum of the solar cycle. This was a moon-down period and the selected orbits were over open-ocean so that the TIP data were not contaminated by visible light from below the ionosphere. The orbits and the trajectory of the tangent point locations for the lines-of-sight are also indicated in Fig. 3. The occultations occur near the orbit plane but are not coincident with the orbit plane. Figure 4 shows the TIP radiances plotted as a function of geomagnetic latitude with the GPS occultation geometry overplotted. In this type of projection, Earth's surface is flat and the normally straight GPS linesof-sight become curves. The Appelton anomalies are clearly indicated by the regions of enhanced emission northward and southward of the geomagnetic equator. The smooth curves indicated by the "diamonds" in the two panels are the minimum ray height trajectories for the GPS occultations. Note that the tangent point of the occultation in the western Pacific (orbit near $171^{\circ} \mathrm{E}$ ) occurs between the anomalies while the occultation for the orbit near $135^{\circ} \mathrm{W}$ occurs immediately northward of the northern anomaly crest. These are ideal test cases for the tomographic inversions.

We will first concentrate on the inversion from the orbit near $135^{\circ} \mathrm{W}$. Figure 5 shows the results of the inversions. Figure 5a shows the measured GOX TEC and the fit to the data. The agreement is very good. Figure $5 \mathrm{~b}$ shows the measured TIP radiances and the fit; again the fit is excellent. Figure $5 \mathrm{c}$ shows a contour plot of the two-dimensional electron density distribution near the anomalies. Note that the peak height of the ionosphere does not change with latitude. This is because the tomographic algorithm assumes that the altitude distribution of the electrons is constant with latitude, which is not physically correct. The peak altitude, $h m F 2$, is known to vary in the equatorial region because the plasma is lifted upward during the daytime by the $\mathbf{E} \times \mathbf{B}$ drift due to the 
electric field generated by the E-region dynamo. As the E-region dynamo is confined to a narrow latitude region surrounding the dip equator, the electric field eventually goes to zero at higher altitudes and the uplift ceases allowing the plasma to drift toward both poles under the influence of gravity and pressure gradients to form the Appelton anomaly. The strength of the plasma uplift is also affected by the pre-reversal enhancement of the zonal electric field. Recent studies also indicate that tidal influences affect the strength of the E-region dynamo (Immel et al. 2006). The effects of all of these factors combined integrated throughout the day determine the vertical distribution of plasma in the low-latitude ionosphere and hence affect the peak height, $h m F 2$. But using a single occultation in the inversion, it is not possible to adequately account for the variation of peak height in this dynamic region of the ionosphere, even if a low order parameterization of the peak height with latitude is included. In our previous studies, we demonstrated that the electron density profile shape in the vicinity of the occultation is accurately determined and that the overall error in the reconstructed electron densities increases with distance from the tangent point trajectory. The increased error is largely due to the algorithm's inability to capture the variation of the peak height with distance away from the profile

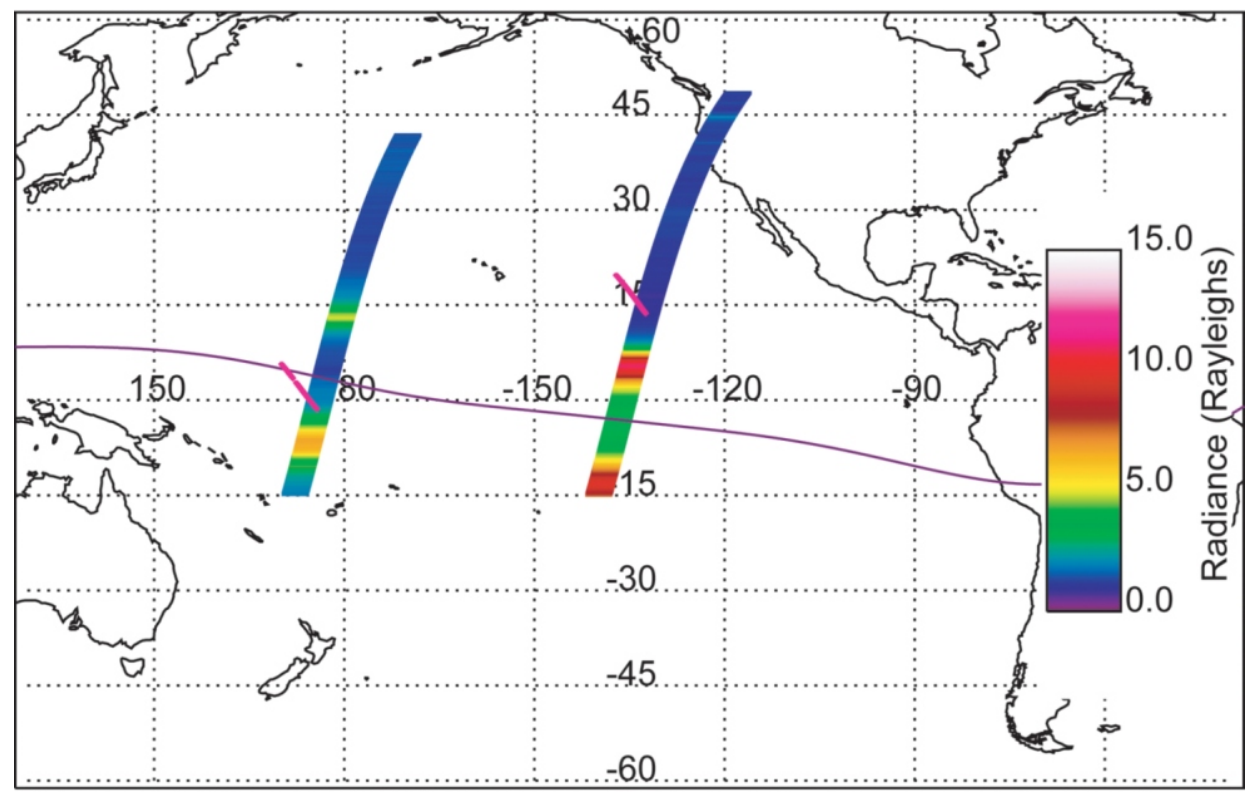

Fig. 3. TIP radiances and GPS occultations used in study. This figure shows the TIP along track radiances shown in color with the tangent point trajectories of the GOX occultations shown as the solid lines.
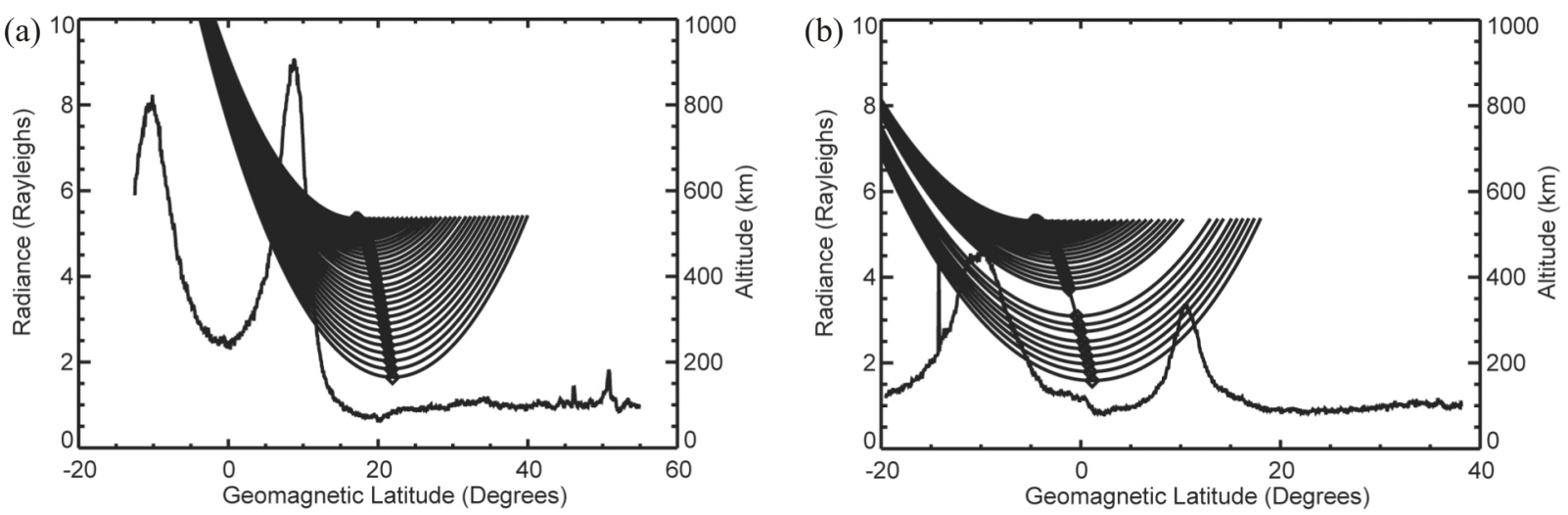

Fig. 4. TIP Radiances and GOX viewing geometries. (a) shows the TIP radiance for the orbit at $135^{\circ} \mathrm{W}$ with the GOX lines-of-sight (curves) overplotted. To improve clarity in this figure, every $10^{\text {th }}$ GOX line-of-sight is shown. The smooth curve indicated by the diamonds is the tangent point trajectory. This occultation occurred slightly northward of the Appelton anomalies. (b) shows the TIP radiance for the orbit at $171^{\circ} \mathrm{E}$ with the GOX lines-of-sight (curves) overplotted. The smooth curve indicated by the diamonds is the tangent point trajectory. This occultation occurred between the Appelton anomalies. 
(a)

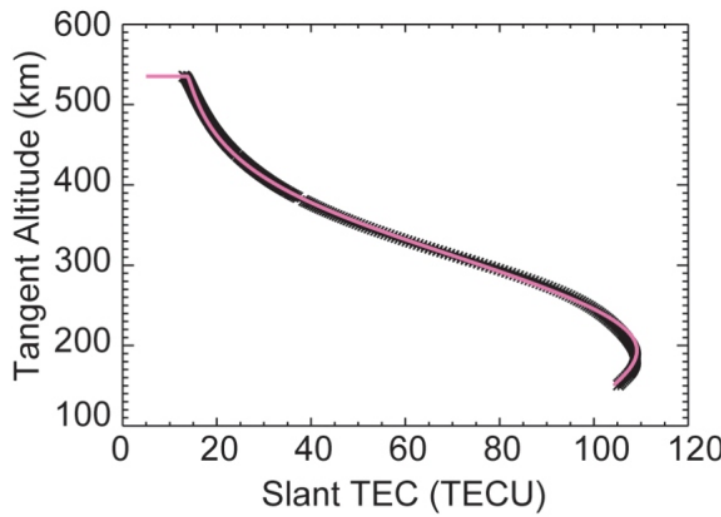

(c)

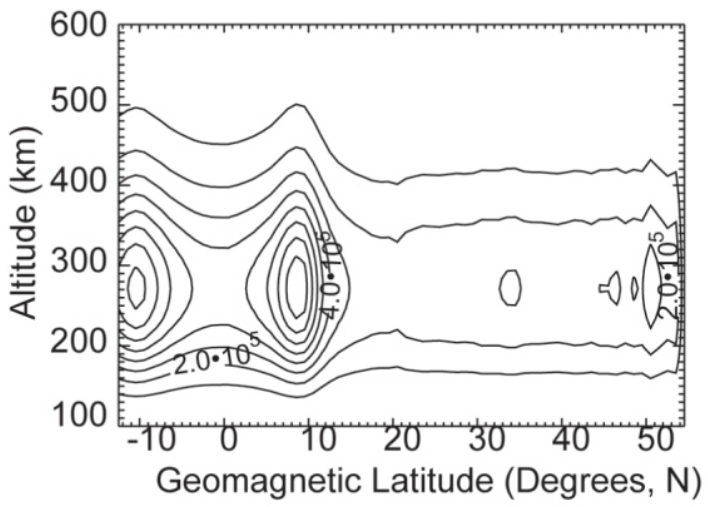

(b)

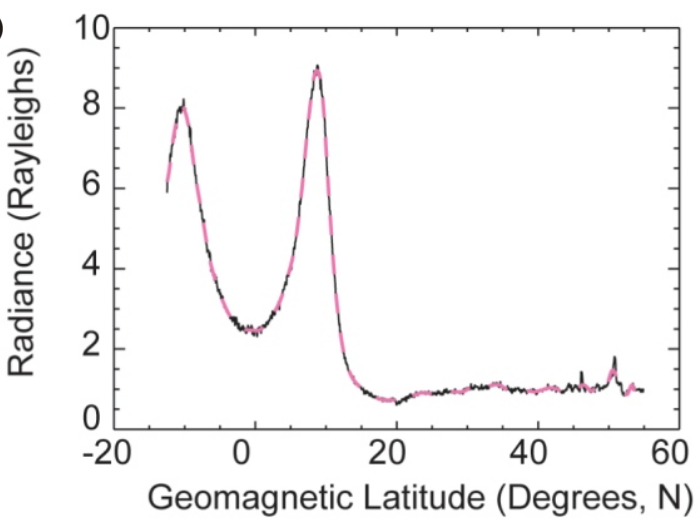

(d)

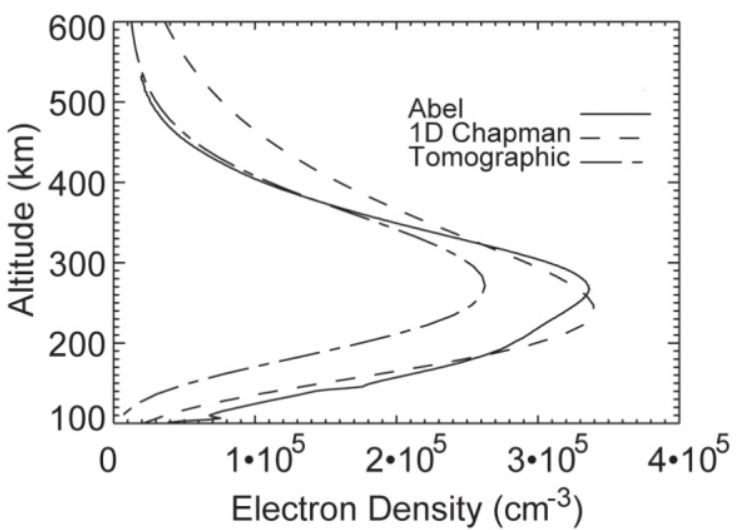

Fig. 5. Inversion results for occultation at $135^{\circ} \mathrm{W}$. (a) shows the GOX TEC and the fit from the inversion (pink line). (b) shows the TIP radiance and the fit (pink dashed line). (c) shows the two-dimensional electron density field reconstructed from the data; the contours are spaced at every $1 \times 10^{5}$ $\mathrm{cm}^{-3}$. (d) shows the electron density profile comparison. The electron density profiles are at a geomagnetic latitude of $\sim 22^{\circ} \mathrm{N}$, as indicated by the diamonds in Fig. 4a.

tangent point trajectory. Figure $5 \mathrm{~d}$ shows the comparison of the vertical electron density profile derived from the tomographic technique, from direct Abel inversion, and from a one-dimensional inversion where spherical symmetry is assumed and a five parameter Chapman layer profile is fit to the TEC data. The 1D Chapman fit yielded an $n m F 2$ (peak electron density) of $3.4 \times 10^{5} \mathrm{~cm}^{-3}$, an $h m F 2$ (peak height) of $241.6 \mathrm{~km}$, and a plasma scale height of $130.6 \mathrm{~km}$. The tomographic inversion yielded an $n m F 2$ of $2.7 \times 10^{5} \mathrm{~cm}^{-3}$, an $h m F 2$ of $271.5 \mathrm{~km}$, and a plasma scale height of $136.0 \mathrm{~km}$. The Abel inversion yielded an $n m F 2$ of $3.4 \times 10^{5} \mathrm{~cm}^{-3}$ and an $h m F 2$ of $272 \mathrm{~km}$. The Abel inversion $n m F 2$ differed from the tomographic solution by $1 \mathrm{~km}$, within the errors of the two inversions. However, the Abel inversion overestimated the peak electron density by $\sim 26 \%$. Furthermore, this overestimate is consistent with the behavior seen in Fig. 1, where the Abel inversion overestimates the radiance just northward of the Appelton anomalies. This is caused by the GOX lines-of-sight intersecting the topside of the Appelton anomalies (Fig. 4a) and therefore observing TEC that is higher because of the electron density gradient. The agreement of the peak heights is fortuitous as the shape of the electron density profile shows what appears to be a layer in the bottomside that is most likely due again to the GOX viewing the northern anomaly crest beyond the tangent point (background density). The 1D Chapman inversion produced an $n m F 2$ that was $\sim 26 \%$ higher than the tomographic inversion. The peak height retrieved by the 1D Chapman inversion is $29.9 \mathrm{~km}$ below the $h m F 2$ from the tomographic inversion. In this case, the intersection of the GOX linesof-sight with the northern anomaly crest "added" TEC to the occultation profile on the bottomside, which made the profile appear to be shifted to lower altitudes. The 1D Chapman inversion is presented to show that it too does not correctly capture the electron density profile and that its flaws are similar to those of the Abel inversion and not due to the Chapman layer formalism. This is necessary as the second tomographic inversion we present does not have a direct Abel inversion available due to a GOX data drop-out, so we compare the tomographic results with those of a 1D Chapman inversion.

We will now discuss the results of the inversion from the orbit near $171^{\circ} \mathrm{E}$. Figure 6 shows the results of the inversions. Figure 6 a shows the measured GOX TEC and the fit to 
(a)

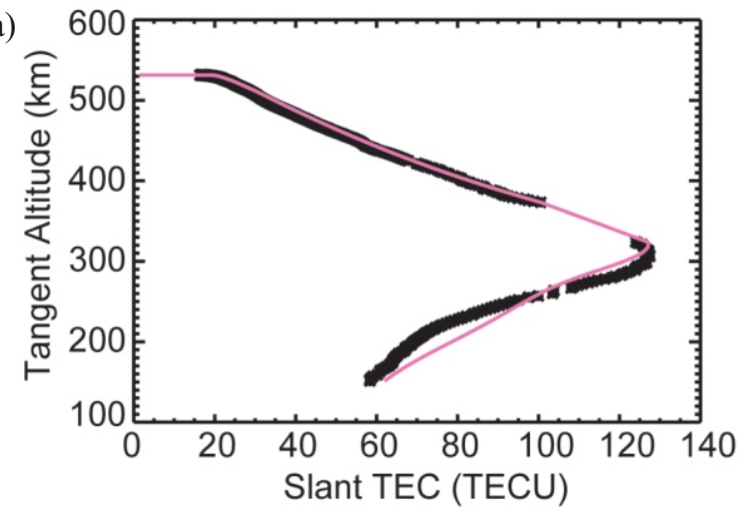

(c)

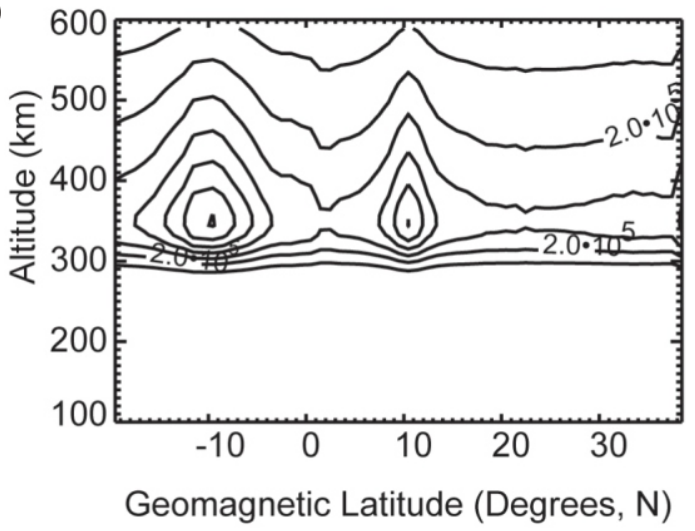

(b)

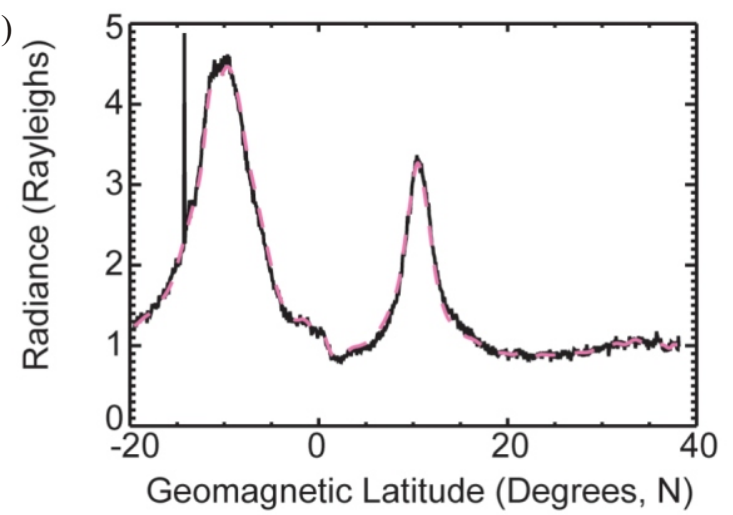

(d)

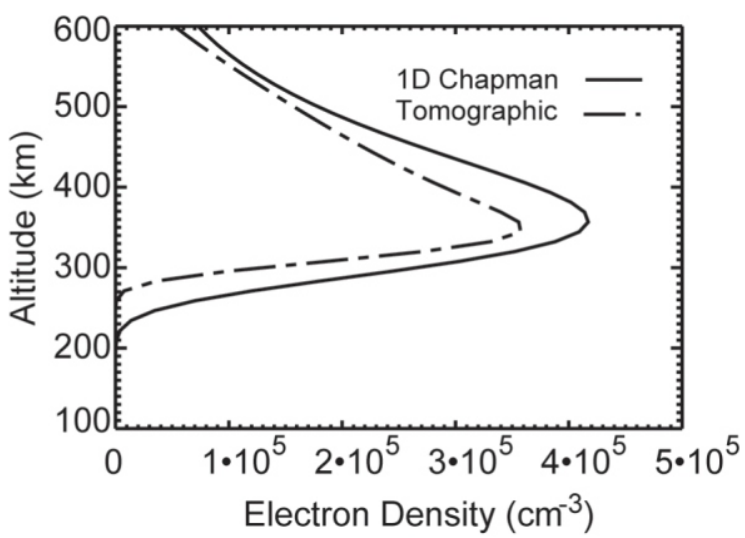

Fig. 6. Inversion results for occultation at $171^{\circ} \mathrm{E}$. (a) shows the GOX TEC and the fit from the inversion (pink line). Note that the TEC fit is not as tight as the fit for $135^{\circ} \mathrm{W}$. (b) shows the TIP radiance and the fit (pink dashed line). (c) shows the two-dimensional electron density field reconstructed from the data; the contours are spaced at every $1 \times 10^{5} \mathrm{~cm}^{-3}$. (d) shows the electron density profile comparison; the Abel TEC was not available as there were data drop-outs that precluded that profile from being routinely inverted. The electron density profiles are at a geomagnetic latitude of $\sim 0^{\circ} \mathrm{N}$, as indicated by the diamonds in Fig. $4 \mathrm{~b}$.

the data. The agreement is not as compelling as it was at $135^{\circ} \mathrm{W}$. Figure $6 \mathrm{~b}$ shows the measured TIP radiances and the fit; again the fit is excellent. Figure $6 \mathrm{c}$ shows a contour plot of the two-dimensional electron density distribution near the anomalies. Note that the peak height of the ionosphere is much higher than it was near $135^{\circ} \mathrm{W}$. Also, the bottomside of the electron density distribution is much sharper than it was at $135^{\circ} \mathrm{W}$. In this sector, ionospheric bubbles and GPS scintillation were observed. The stronger gradients on the bottomside may be indicative of a precursor for bubble formation. Note that due to the assumption of a constant altitude profile that is used in our algorithm, the high peak height $(h m F 2)$ and sharp bottomside fall-off are extended to all latitudes. This may not be realistic as the physical conditions that are likely causing the sharp bottomside fall-off may not extend for great distances north and south. The sharp bottomside fall-off and high peak height are most accurate in the region where the tangent point trajectory of the GOX line-of-sight passes.

Figure $6 \mathrm{~d}$ shows the comparison of the vertical electron density profile derived from the tomographic technique, from direct Abel inversion, and from a one-dimensional in- version where spherical symmetry is assumed and a five parameter Chapman layer profile is fit to the TEC data. The 1D Chapman fit yielded an $n m F 2$ (peak electron density) of $4.2 \times 10^{5} \mathrm{~cm}^{-3}$, an $h m F 2$ of $358.9 \mathrm{~km}$, and a plasma scale height of $107.6 \mathrm{~km}$. The tomographic inversion yielded an $n m F 2$ of $3.4 \times 10^{5} \mathrm{~cm}^{-3}$, an $h m F 2$ of $349.3 \mathrm{~km}$, and a plasma scale height of $64.8 \mathrm{~km}$. There was no Abel inversion as the large data drop-out just above the peak of the TEC profile (Fig. 6a) precluded inversion through the entire profile. The 1D Chapman fit produced an $n m F 2$ that was $\sim 24 \%$ higher than the tomographic solution. The occultation occurred between the anomaly crests so both crests contributed enhancements to the TEC that could not be accommodated for in a simple 1D, spherically symmetric, inversion. The peak height retrieved by the 1D Chapman inversion is $9.6 \mathrm{~km}$ below the $h m F 2$ from the tomographic inversion, within the errors of the inversions. In this case, the intersection of the GOX lines-of-sight with both anomaly crests "added" TEC to the occultation profile throughout the profile, which increased the overall TEC and therefore the peak electron density but preserved the shape with altitude so that the peak height was not strongly affected. The tomographic inversion 
also retrieved the sensitivity of the TIP instrument. The sensitivity was $621 \mathrm{ct} \mathrm{s}^{-1}$ Rayleigh $^{-1}$ for the orbit near $135^{\circ} \mathrm{W}$ and $745 \mathrm{ct} \mathrm{s}^{-1}$ Rayleigh $^{-1}$ for the orbit near $171^{\circ} \mathrm{E}$. These results differ as uncertainties on the scale height and $n m F 2$ affect the retrieved TIP intensity, which affects the value of the retrieved sensitivity. We average these values together to get $683 \mathrm{ct} \mathrm{s}^{-1}$ Rayleigh $^{-1}$. The estimated pre-flight value of the sensitivity for TIP instruments was approximately $500 \mathrm{ct}$ $\mathrm{s}^{-1}$ Rayleigh $^{-1}$. Though the two values differ by $\sim 37 \%$, we feel that the value estimated on-orbit is the more accurate of the two values.

Figure 7 shows the variation of the retrieved $n m F 2$ with geomagnetic latitude for the two orbits considered in this work. Figure 6a shows the tomographic inversion fit to the GOX TEC. This is not a high quality fit. The anomaly crests are more symmetric near $135^{\circ} \mathrm{W}$ than they are at $171^{\circ} \mathrm{E}$. The northern anomaly is depressed relative to the southern anomaly and the locations of the crests are not symmetric with respect to the dip equator. This asymmetry suggests that the reason that the algorithm produced a poor fit is that ionosphere sampled by the GOX occultation differed from that observed by the TIP. A latitudinal asymmetry of the anomalies can be caused by a meridional F-region wind blowing in the northerly direction which would cause plasma to "pile up" in the southern anomaly crest and to be blown down the field lines in the northern hemisphere away from the northern anomaly crest where it would recombine more rapidly. This would also cause the latitudes of the crests in the two hemispheres to differ. However, the shapes of the southern anomaly crests in Fig. 7 for the two orbits are very similar suggesting that the conditions affecting the anomaly crests in the two regions are similar. Thus, the northern crests should also have a similar shape if trans-equatorial winds were the cause of the asymmetry, yet the shapes of the northern anomalies are different. Another explanation lies in

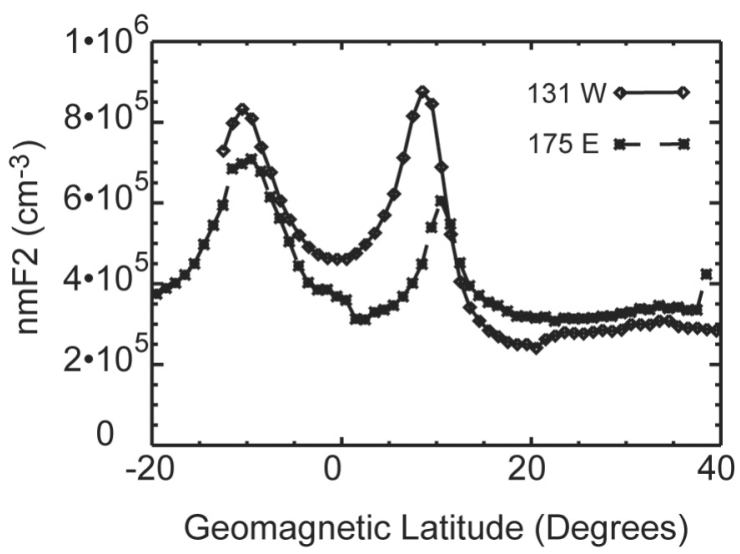

Fig. 7. $n m F 2$ comparison. The variations of the peak density with geomagnetic latitude are shown for the two inversions. The $n m F 2$ derived for $171^{\circ} \mathrm{E}$ shows some structure that is not present in the $n m F 2$ variation at $135^{\circ} \mathrm{W}$. This is most likely due to bubble formation in the Kwajelein sector that degraded the quality of the TEC fit in Fig. 6a. the non-conjugacy of the observations of the northern and southern anomaly crests. The FORMOSAT-3/COSMIC satellites are in a $72^{\circ}$ inclination orbit, which causes most of the orbits to run across magnetic field lines. Thus, the sampling of the anomaly crests is always non-conjugate. This non-conjugacy can mean that the TIP sensor is seeing plasma distributions in the two anomalies that are from different flux tubes. If a plasma depletion ("bubble") has occurred along one flux tube it may not be present in another nearby flux tube. So, the difference in the two anomaly crests might be more indicative of a depletion extending across the equator on a flux tube seen by the TIP in the northern anomaly while the flux tube observed by the TIP in the southern anomaly might show no depletion (see Coker et al. 2009; Chua et al. 2009). The strong bottomside density gradient seen near $171^{\circ} \mathrm{E}$ (Figs. 6c and d) supports the assertion that an ionospheric bubble was observed by the FORMOSAT-3/COSMIC sensors; this also can explain the poor fit to the occultation data as the GOX and TIP sensors observed an ionosphere with longitudinal structure. Observations made using all-sky cameras on Kwajalein Atoll on 14 and 17 September 2006 indicate the presence of multiple bubbles and associated spread-F (Coker et al. 2009).

\section{CONCLUDING REMARKS}

We presented inversions of the TEC measured by the GPS Occultation Experiment (GOX) and the Tiny Ionospheric Photometer (TIP) on FORMOSAT-3/COSMIC satellite \#6 on 15 September 2006. The two tomographic inversions from near the Appelton anomalies, in a region of large electron density gradients, were compared with Abel inversions and 1D Chapman inversions and demonstrated that the tomographic inversions differed from the Abel and 1D Chapman inversions as expected. We demonstrate that the spherically symmetric Abel and 1D Chapman inversions overestimate the $n m F 2$ by $\sim 30 \%$, when compared to the tomographic inversions, and that these overestimates are consistent with the GOX viewing geometry. The TIP sensitivity is also determined as a byproduct of the inversion and is shown to be consistent with pre-flight estimates. This demonstrates a new capability to calibrate the TIP instruments on-orbit.

Overall, our improved algorithm does a good job of retrieving the electron density from the GPS TEC's and the photometer radiances. The technique permits an accurate retrieval of the ionosphere over a range of latitude in areas with high gradients. Clearly, further testing, evaluation, and validation is needed, but the promise of the tomographic technique has been demonstrated. The next step in development of the algorithm is to account for the variation of peak height with latitude. There are two possible ways to do this. One way is to add in information regarding the variation of the peak height with latitude from models perhaps using the 
Similarity Transform approach of Meier et al. (2001). Another way is to extend the tomographic algorithm to ingest multiple occultations and invert them simultaneously with the TIP radiance data. Given the factors that can affect the peak height in the equatorial region, extending the tomographic algorithm to ingest and invert additional occultation profiles offers the greatest promise. An alternative approach, based on the Compensated TEC technique (Tsai et al. 2009), also shows promise in better resolving the electron density distribution in the equatorial ionosphere.

The FORMOSAT-3/COSMIC constellation is expected to produce $\sim 2500$ occultations per day, many of these will be near the orbit plane on the nightside where our algorithm works well.

Acknowledgments This work was supported by the FORMOSAT-3/COSMIC Program at the University Corporation for Atmospheric Research and by the Office of Naval Research. We are also grateful to the National Space Office (NSPO) of the Republic of China (Taiwan) for supporting the development of the TIP sensors.

\section{REFERENCES}

Barth, C. A. and S. Schaffner, 1970: OGO 4 spectrometer measurements of the tropical ultraviolet airglow. J. Geophys. Res., 75, 4299-4306, doi: 10.1029/JA075i022p04299. [Link]

Bevington, P. R., 1969: Data Reduction and Error Analysis for the Physical Sciences, McGraw-Hill, New York, NY.

Chakrabarti, S., R. Kimble, and S. Bowyer, 1984: Spectroscopy of the EUV (350-1400 $\AA$ ) nightglow. J. Geophys. Res., 89, 5660-5664, doi: 10.1029/JA089iA07p05660. [Link]

Chamberlain, J. W. and D. M. Hunten, 1987: Theory of Planetary Atmospheres: An Introduction to Their Physics and Chemistry, Academic Press, Inc., Orlando, FL.

Chandra, S., E. I. Reed, R. R. Meier, C. B. Opal, and G. T. Hicks, 1975: Remote sensing of the ionospheric $F$ layer by use of O I 6300- $\AA$ and O I 1356- $\AA$ observations. J. Geophys. Res., 80, 2327-2332, doi: 10.1029/JA080i016p 02327. [Link]

Chua, D. H., K. F. Dymond, S. A. Budzien, C. Coker, and J. Y. Liu, 2009: Horizontal ionospheric electron density gradients observed by FORMOSAT-3/COSMIC TIP: Spatial distributions and effects on VLF wave propagation at mid-latitudes. Terr. Atmos. Ocean. Sci., 20, 251-259, doi: 10.3319/TAO.2008.01.16.01(F3C). [Link]

Coker, C., K. F. Dymond, S. A. Budzien, D. H. Chua, J. Y. Liu, D. N. Anderson, S. Basu, and T. R. Pedersen, 2009: Observations of the ionosphere using the Tiny Ionospheric Photometer. Terr. Atmos. Ocean. Sci., 20, 227-235, doi: 10.3319/ TAO.2008.01.18.02(F3C). [Link]

Dymond, K. F. and R. J. Thomas, 2001: A technique for using measured ionospheric density gradients and GPS occultations for inferring the nighttime ionospheric electron density. Radio Sci., 36, 1141-1148, doi: 10.1029/2000RS 002430. [Link]

Dymond, K. F., S. E. Thonnard, R. P. McCoy, and R. J. Thomas, 1996: A Technique for Determining F Region Electron Densities Using Optical Measurements of Recombination Radiation. Proc. 1996 Ionospheric Effects Symposium, 7-9 May 1996, Alexandria, VA., 346-353.

Dymond, K. F., S. E. Thonnard, R. P. McCoy, and R. J. Thomas, 1997: An optical remote sensing technique for determining nighttime F region electron density. Radio Sci., 32, 19851996, doi: 10.1029/97RS01887. [Link]

Dymond, K. F., J. B. Nee, and R. J. Thomas, 2000: The tiny ionospheric photometer: An instrument for measuring ionospheric gradients for the FORMOSAT-3/COSMIC constellation. In: Applications of Constellation Observing System for Meteorology, Ionosphere, and Climate, Springer, New York, NY, 273-290.

Feldman, P. D., A. F. Davidsen, W. P. Blair, C. W. Bowers, S. T. Durrance, G. A. Kriss, H. C. Ferguson, R. A. Kimble, and K. S. Long, 1992: The spectrum of the tropical oxygen nightglow observed at $3 \AA$ resolution with the hopkins ultraviolet telescope. Geophys. Res. Lett., 19, 453-456, doi: 10.1029/92GL00376. [Link]

Hajj, G. A., R. Ibañez-Meier, E. R. Kurzinski, and L. J. Romans, 1994: Imaging the ionosphere with the global positioning system. Int. J. Imaging Syst. Technol., 5, 174-184, doi: 10.1002/ima.1850050214. [Link]

Hicks, G. T. and T. A. Chubb, 1970: Equatorial Aurora/Airglow in the Far Ultraviolet. J. Geophys. Res., 75, 6233-6248, doi: 10.1029/JA075i031p06233. [Link]

Immel, T. J., E. Sagawa, S. L. England, S. B. Henderson, M. E. Hagan, S. B. Mende, H. U. Frey, C. M. Swenson, and L. J. Paxton, 2006: Control of equatorial ionospheric morphology by atmospheric tides. Geophys. Res. Lett., 33, L15108, doi: 10.1029/2006GL026161. [Link]

Kalmanson, P. C., S. A. Budzien, C. Coker, and K. F. Dymond, 2004: The tiny ionospheric photometer instrument design and operation. Proc. SPIE, 5660, 259, doi: 10.1117/12. 578341. [Link]

Meier, R. R., 1991: Ultraviolet Spectroscopy and remote sensing of the upper atmosphere. Space Sci. Rev., 58, doi: 10.1007/BF01206000. [Link]

Meier, R. R., J. M. Picone, D. P. Drob, and R. G. Roble, 2001: Similarity transformation-based analysis of atmospheric models, data, and inverse remote sensing algorithms. $J$. Geophys. Res., 106, No. A8, 15519-15532, doi: 10.1029/ 2001JA000062. [Link]

Melendez-Alvira, D. J., R. R. Meier, J. M. Picone, P. D. Feldman, and B. M. McLaughlin, 1999: Analysis of the oxygen nightglow measured by the hopkins ultraviolet telescope: Implications for ionospheric partial radiative recombination rate coefficients. J. Geophys. Res., 104, 14901-14913, doi: 10.1029/1999JA900136. [Link]

Menke, W., 1989: Geophysical Data Analysis: Discrete Inverse Theory, International Geophysics Series, No. 45, Aca- 
demic Press, San Diego.

Picone, J. M., R. R. Meier, O. Kelley, K. F. Dymond, R. J. Thomas, D. J. Melendez-Alvira, and R. P. McCoy, 1997: Investigation of ionospheric $\mathrm{O}^{+}$remote sensing using the 834-Å airglow. J. Geophys. Res., 102, 2441-2456, doi: 10.1029/96JA03314. [Link]

Press, W. H., B. P. Flannery, S. A. Teukolsky, and W. T. Vetterling, 1992: Numerical Recipes: The Art of Scientific Computing, Cambridge University Press, New York, NY.

Samson, J. A. R., 1967: Techniques of Vacuum Ultraviolet Spectroscopy, Pied Publications, Lincoln, Nebraska.

Tikhonov, A. N., 1963: Solution of incorrectly formulated problems and the regularization method. Dokl. Akad. Nauk SSSR, 151, 501-504.
Tinsley, B. A. and J. A. Bittencourt, 1975: Determination of F region height and peak electron density at night using airglow emissions from atomic oxygen. J. Geophys. Res., 80 , 2333-2337, doi: 10.1029/JA080i016p02333. [Link]

Tinsley, B. A., A. B. Christensen, J. Bittencourt, H. Gouveia, P. D. Angreji, and H. Takahashi, 1973: Excitation of oxygen permitted line emissions in the tropical nightglow. J. Geophys. Res., 78, 1174-1186, doi: 10.1029/JA078i007p 01174. [Link]

Tsai, L. C., C. H. Liu, and T. Y. Hsiao, 2009: Profiling of ionospheric electron density based on FormoSat-3/COSMIC data: Results from the intense observation period experiment. Terr. Atmos. Ocean. Sci., 20, 181-191, doi: 10.3319/ TAO.2007.12.19.01(F3C). [Link] 